\title{
DIABETES; A HINDRANCE IN MUSCULOSKELETAL DISEASES
}

\author{
Blessy. K. Johnson*, Mareena Raju, Ambarish S, Bharathii D R
}

SJM College of Pharmacy, Chitradurga-577502

Article Info: Received 28 April 2019; Accepted 23 May. 2019

DOI: https://doi.org/10.32553/jbpr.v8i3.602

Address for Correspondence: Blessy. K. Johnson, Pharm D Intern, SJM College of Pharmacy, Chitradurga-577502

Conflict of interest statement: No conflict of interest

\section{ABSTRACT:}

Background: Diabetes mellitus (DM) affects connective tissues in many ways and causes different alterations in periarticular and skeletal systems. Musculoskeletal complaints (MSCs) are among the major health problems worldwide and the most frequent cause of long term sickness.

Objectives: To assess the prevalence, treatment pattern of musculoskeletal diseases with diabetes mellitus and to evaluate the impact of patient counselling overcome the barriers of diabetes in musculoskeletal disease.

Materials \& Methods: A prospective observational study was carried out for a period of six months at Departments of Orthopedic and General medicine, Basaveshwara Medical College \& Research center and Ambarish's Clinic,Chithradurga. A total of 118 subjects were included as per study criteria.

Results: Among 118 subjects 17 (14.4\%) had musculoskeletal manifestations with DM. The study shows that $55.9 \%$ patients were males and $44.06 \%$ were females. The maximum number of patients comes under the age of 51-60yrs. The NSAIDs prescribed were Diclofenac (8.69\%), Paracetamol (8.69\%) and Piroxicam 4(8.69\%) and the Antidiabetic drugs in present study were Oral hypoglycemic followed by Insulin. Most of the patients are affected with Osteoarthritis (47.05\%), followed by Adhesive capsulitis $(11.76 \%)$ and Osteoporosis (11.76\%).

Conclusion: The study reveals that the prevalence of musculoskeletal disease with DM is significantly high and osteoarthritis is the common disease. Males are more affected than females with an age group of 51-60.

Key words: Diabetes mellitus, musculoskeletal disease, prevalence, prescription pattern.

\section{INTRODUCTION}

Diabetes mellitus is a group of disorders characterized by chronic hyper glycaemia due to relative insulin deficiency, or resistance or both $^{1}$.According to world health organization (WHO) statistics published in 2013, approximately 347 million people worldwide suffer from diabetes, and by 2023diabetes will be the seventh leading cause of death ${ }^{2}$. India is popularly known as a "diabetes capital of the world". ${ }^{3}$ The causes of increased musculoskeletal pain in people with diabetes are probably related to vascular insufficiency, neuropathy, decreased insulin-like growth factor, accelerated osteoporosis, obesity sedentary life style and other factors. ${ }^{2}$
DM affects connective tissues in many ways and causes different alterations in periarticular and skeletal systems. Several musculoskeletal disorders have been described, which can be divided into three categories: A) Disorders which represent intrinsic complications of diabetes, such as limited joint mobility or diabetic cheiroarthropathy, stiff hand syndrome, and diabetic muscular infraction. B) Disorders with an increased incidence among diabetics, such as Dupuytren's disease, Shoulder capsulitis, neuropathic arthropathy, osteopenia(in type 1 DM),Flexor Tenosynovitis, septic arthritis, acute proximal neuropathy, proximal motor neuropathy, Pyomyositis and the diffuse idiopathic skeletal Hyperostosis(DISH) syndrome, Backache .

C) Disorders for which a possible association with diabetes has been proposed but not proven yet, 
such as osteoarthritis and the carpal tunnel syndrome. ${ }^{4}$

Rheumatic disorders in DM have been associated with disease duration, degree of metabolic control and the presence of end organ damage. ${ }^{5}$

Non -steroidal anti-inflammatory drugs (NSAID's) are widely prescribed for the treatment of pain and inflammation in patients with various musculoskeletal. ${ }^{6}$ Most currently available traditional NSAIDs act by inhibiting COX enzyme. The inhibition of COX-2 is thought to mediate, the antipyretic, analgesic, and anti-inflammatory actions of traditional NSAIDs, while the simultaneous inhibition of cyclooxygenase-1(COX1). ${ }^{7}$ The oral Anti-diabetic drug classes were Biguanides, Sulfonylureas, Alpha-Glucosidase Inhibitor, Repaglinide and Thiazolidinediones. Metformin was the only prescribed Biguanides. Insulin was prescribed for patients with type 2 Diabetes mellitus. ${ }^{8}$

Co-morbidity among patients with diabetes is associated with considerable consequences for health care and related costs. ${ }^{9}$ Management of every patient should commence with a detailed assessment at the initial diagnosis including an appraisal of diabetes complications and risk factors for complications. This provides the basis for continuing care that includes a treatment plan, treatment administration, monitoring, and review. ${ }^{10}$

Health promotion has been described as, the process of enabling people to increase control over, and to improve, their health. There are five strategies to improve better patient care: Build healthy public policy, Create supportive environments, Strengthening community action, Developing personal skills, Re-orienting health services. ${ }^{11}$

\section{MATERIALS AND METHODS}

Study Site: The study was conducted at both outpatient and inpatient Departments of Orthopedic and General medicine of Basaveshwara Medical college Hospital \& Research Center and Ambarish's Health center, Chitradurga.

Study Design: A prospective Interventional study.

Study Period: This study was conducted for 6 months from 2017 to 2018.
Study Subjects: This study included inpatients and outpatients visiting Orthopedic as well as General Medicine departments. Patients who meet the following criteria were enrolled.

\section{STUDY CRITERIA}

\section{Inclusion Criteria}

- Inpatients and outpatients of Orthopedic and Medicine departments.

- $\quad$ Subjects of both genders.

- Subjects who were diagnosed with any musculoskeletal disorder and Type 2 Diabetes mellitus for more than 2 years.

- $\quad$ Subjects who were above 30 years.

Exclusion Criteria

- Pregnant and lactating women.

- Subjects who met with accident as well as fractures.

- $\quad$ Subjects who were at Coma stage.

\section{STATISTICAL ANALYSIS}

The collected data was entered in Microsoft Excel 2010 version and Descriptive method was used for the analysis.

\section{RESULTS}

\section{Distribution of subjects according to age.}

Out of 118 patients, maximum number of patients comes under the age of $51-60$ and minimum number of patients comes under the age of 81-90.

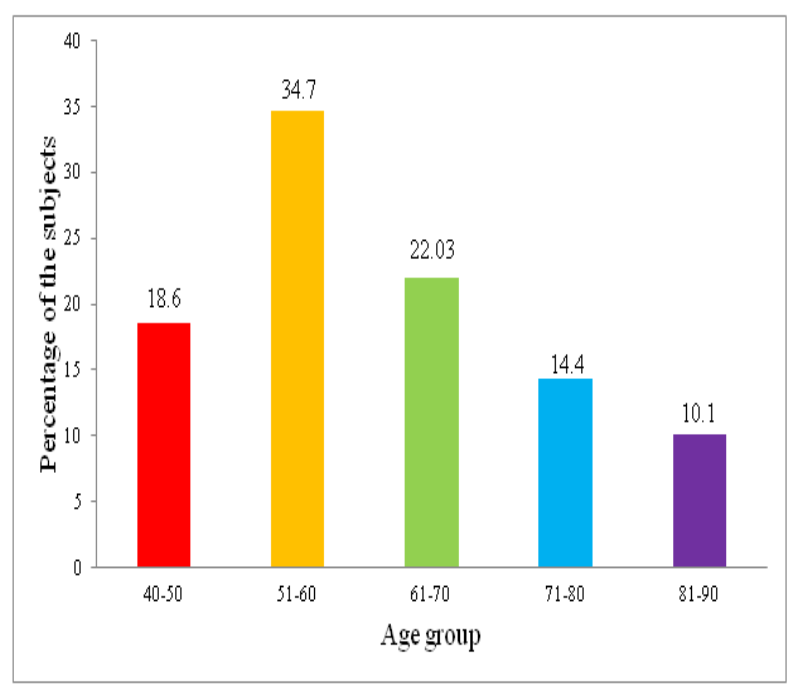

Figure 1: Age group distribution

2. Distribution of subjects according to Gender. 
Out of 118 patients $55.9 \%$ were males and $44.06 \%$ were females.

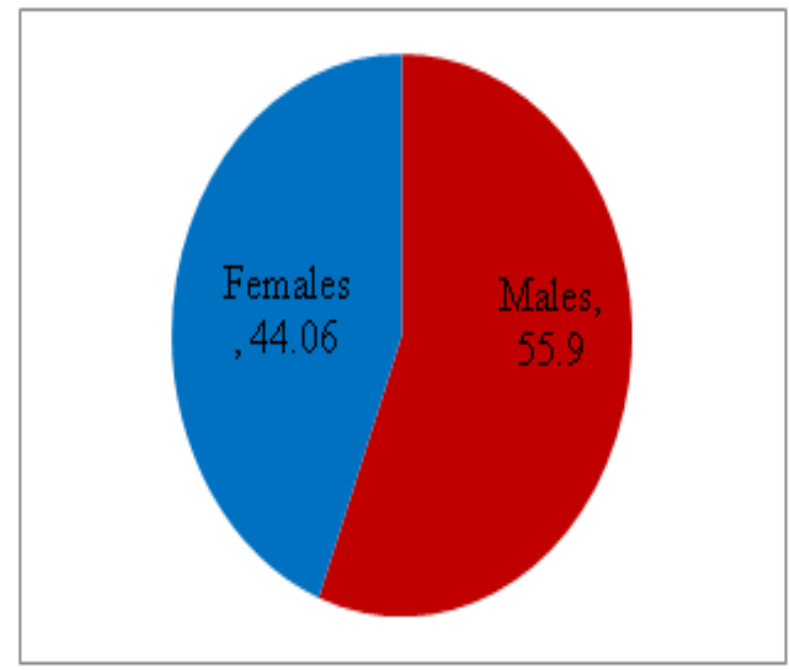

Figure 2: Gender distribution

\section{Distribution of subjects according to diagnosis}

Out of 118 Patients $14.4 \%$ patients were suffering from diabetes with musculoskeletal disease and 101 with Diabetes.

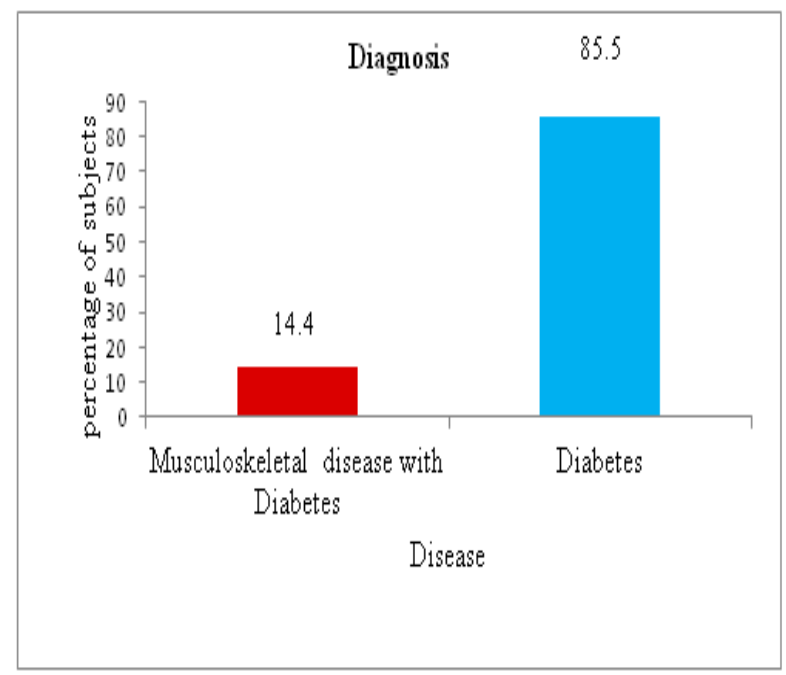

Figure: 3 Distribution of subjects according to diagnosis

4. Distribution of subjects according to Musculoskeletal Diseases.

Out of 17 patients, 8 patients were suffering with OA knee, 2 patients with adhesive capsulitis and osteoporosis, 1 patient with Rheumatoid arthritis, Trapazious muscle spasm, Lumbar Spondylosis, Reflex sympathetic dystrophy and diabetic peripheral neuropathic pain.

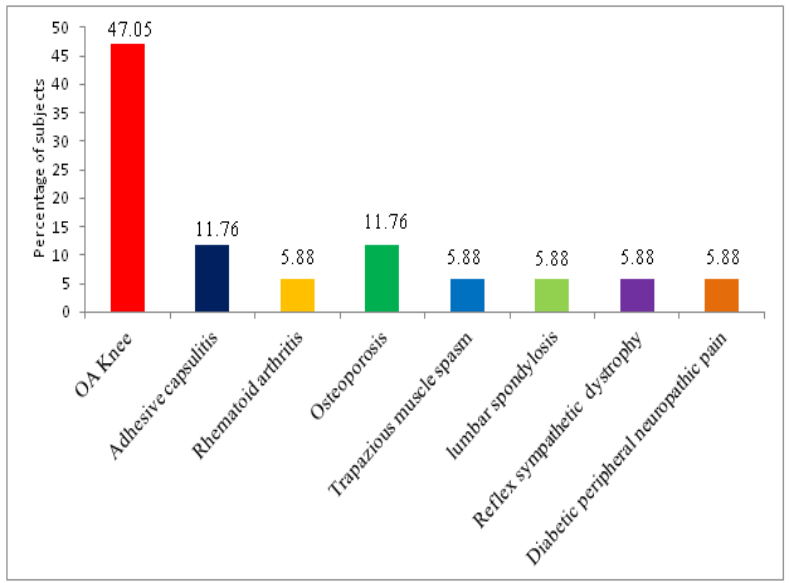

Figure 4: Differential diagnosis

\section{Distribution of Drugs according to class}

Out of 81 drugs prescribed, oral analgesics 46 were the drugs prescribed with high frequency and antibiotics 1 with least frequency.

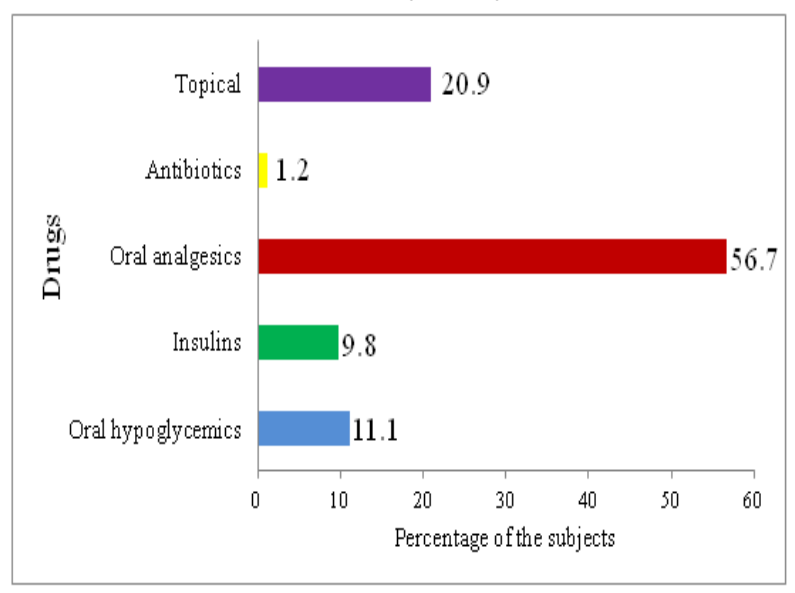

Figure 5: Distribution of drugs according to class

\section{Impact of patient counselling}

During the visit 1 , the subjects were having various problems associated with musculoskeletal disease and diabetes. Patient counselling was done and the patients were reviewed in the subsequent visit which revealed that the knowledge about the disease has improved with reduction in complaints. 11 patients showed improvement in their condition, 4 patients remained the same whereas 2 patients only had slight improvement.

\section{DISCUSSION}

The present prospective interventional study was conducted to assess the prevalence and treatment pattern of musculoskeltal disease associated with diabetes and to assess the impact of patient counselling in the outpatients and inpatients of 
Basaveshwara medical college hospital, Chitradurga. Musculoskeletal (MSK) complications of diabetes mellitus (DM) are the most common endocrine arthropathies. ${ }^{12}$ Several personal, occupational and psychosocial factors are additive in the presence of DM and thus increase the frequency of hand and shoulder problems in the diabetic population. ${ }^{13}$ A comprehensive pharmaceutical care plan for the patient with DM will integrate considerations of goals to optimize blood glucose control and protocols to screen for, prevent, or manage microvascular and macrovascular complications. ${ }^{14}$

In another twin study conducted by Umesh KS et al, a similar result was observed in the total number of subjects assessed for various Musculoskeletal manifestations, subjects amounting to had the manifestation and their age group. ${ }^{3}$ In present study, 118 were included and 17 (14.4\%) had musculoskeletal manifestations with DM.They were divided into five groups based on their age. Out of 118 patients $41(34.7 \%)$ were from 51-60 age group. In the study population , 55.9\% patients were males and $44.06 \%$ were females, a similar result was observed in a study done by Ahmad I et al.,on the topic "Musculoskeltal disorders in long standing Diabetes mellitus cases.1

Among 17 patients, NSAIDs prescribed were Diclofenac (8.69\%), Paracetamol (8.69\%) and Piroxicam 4(8.69\%). (as shown in table no-9) In the study done by Farheen $\mathbf{H}$ et al., on Prescription pattern of non-steroidal anti-inflammatory drugs in patients with acute musculoskeletal pain , the result obtained was Diclofenac followed by Combination of paracetamol and tramadol. ${ }^{7}$ Mostly prescribed Antidiabetic drugs were Oral hypoglycemics followed by Insulin. Oral hypoglycemic prescribed were Glimepiride + Metformin 4(44.4\%), Glimepiride 2(22.3\%), Glipizide + Metformin $2(22.2 \%)$ and metformin $1(11.1 \%)$. (as shown in table no-7)The insulin were Human Actrapid 7(87.5\%), Isophane 1(12.5\%).(as shown in table no8)In the study done by Yusefzadeh $\mathbf{G}$ et al., on Prescription Pattern Study in Type 2 Diabetes Mellitus in Diabetic out Patients in Private Clinics in Iran, the result obtained was Oral antidiabetic drugs were maximally prescribed followed by insulin. ${ }^{8}$

In the study population $47.05 \%$ had Osteoarthritis, followed by Adhesive capsulitis
(11.76\%),Osteoporosis(11.76\%).Attar

SM., conducted a study on Musculoskeletal manifestations in diabetic patients at a tertiary center. ${ }^{12}$ And the results found that the most common manifestations were carpal tunnel syndrome, shoulder adhesive capsulitis and diabetic amyotrophy which is also the pattern seen in another study done by Kidwai SS et al., on Upper limb musculoskeletal abnormalities in type 2 diabetic patients in low socioeconomic strata in Pakistan. ${ }^{13}$

In this present study the knowledge about the disease, effectiveness of therapy, role of selfcare management were assessed by direct interaction with patient and it shows positive response which is further supported by the results of a similar study conducted by Rashid $\mathbf{M}$ et al., on Prevalence of Co-morbidities in Type 2 Diabetes Mellitus Patients, the Awareness Level and the Impact of Pharmacist's Patient Education Program. ${ }^{9}$

\section{CONCLUSION}

According to the analyzed results and from review of literature, the conclusions made are;

1. Prevalence of musculoskeletal disease with diabetes was significantly high.

2. This can be attributed to the prevalence of gender inequality as a result of which males are preferentially taken to tertiary care institutes for treatment as compared to females with a similar severity of illness.

3. Osteoarthritis was most common disease found in musculoskeletal diseases.

4. The optimum management of individual patients may vary in the clinical course of the disease and individual needs.

5. Mostly prescribed Antidiabetic drugs were Oral hypoglycemic followed by Insulin.

6. For musculoskeletal diseases NSAIDs like diclofenac, Paracetamol and piroxicam were most commonly prescribed.

7. The knowledge regarding disease, diet and life style modification among musculoskeletal disorder with diabetes mellitus was improved after the patient education. 
Blessy. K. Johnson et al., Journal of Biomedical and Pharmaceutical Research

\section{ACKNOWLEDGEMENT}

I am highly indebted to my guide Dr.Bharathi D R, Principal SJM College of Pharmacy, Chitradurga, for their continuous support, Dr.Ambarish Sharma for the motivation and guidance throughout the tenure of this work inspite of hectic schedule who truly remain driving spirit and their experience gave me light in this work and helped me in clarifying he abstruse concepts.

\section{REFERENCES}

1. Ahmad I, Nadeem D, Aziz A. Musculoskeletal disorders in long standing Diabetes Mellitus cases. The Journal of Pakistan Orthopedic Association 2008;20(1):38-48.

2. Pai LW, Hung CT, Li SF, Chen LL, Chung YC, Liu $\mathrm{HL}$. Musculoskeletal pain in people with and without type 2 diabetes in Taiwan: a population-based, retrospective cohort study. BMC Musculoskeletal Disorders 2015;16(364)1-7.

3. Umesh KS, Ranganatha YP. Musculoskeletal and Joint Manifestations in Type II Diabetes Mellitus. American Journal of Advanced Drug Delivery 2015;3(1):59-63.

4. Douloumpakas I,Pyrpasopoulou A,Triantafyllou A, Sampanis Ch , Aslanidis S. Prevalence of musculoskeletal disorders in patients with type 2 diabetes mellitus: a pilot study.HIPPOKRATIA 2007;11(4):216-18.

5. Bhat TA, Dhar SA, Dar TA, Naikoo MA, Naqqash MA, Bhat A, Butt MF. The Musculoskeletal Manifestations of Type 2 Diabetes Mellitus in a Kashmiri Population. International Journal of Health Sciences 2016;10(1):57-68.

6. Lanas A, Tom MP, Roncales P, Gonzalez MA , Zapardiel J. Prescription of and Adherence to Non-Steroidal Anti-Inflammatory Drugs and Gastro protective Agents in At-Risk
Gastrointestinal Patients. The American Journal of Gastroenterology 2012;107:707-14.

7. Farheen $H$, Subhani $G$, Mohsin M. Prescription pattern of non-steroidal anti-inflammatory drugs in patients with acute musculoskeletal pain. International Journal of Basic \& Clinical Pharmacology 2016;5(6):2504-09.

8. Yusefzadeh G, Sepehri G, Goodarzi H, Shokoohi M. Prescription Pattern Study in Type 2 Diabetes Mellitus in Diabetic out Patients in Private Clinics in Kerman,Iran. British Journal of Medicine \& Medical Research 2014;4(32):5144-53.

9. Rashid KM, Anandhasayanam A, Kannan $S$, Manohar SD. Prevalence of Co-morbidities in Type 2 Diabetes Mellitus Patients, the Awareness Level and the Impact of Pharmacist's Patient Education Program. International Journal of Pharma Research \& Review 2015;4(5):11-20.

10. Hughes JD, Wibowo $Y$, Sunderland B, Kreshnik Hoti. The role of the pharmacist in the management of type 2 diabetes: current insights and future directions. Integrated Pharmacy Research and Practice 2017;6:1527.

11. Woolcock K. Arthritis - a priority area for action 2011;30:842-47.

12. Attar SM. Musculoskeletal manifestations in diabetic patients at a tertiary center. Libyan J Med 2012;7(19162) :1-7.

13. Kidwai SS, Wahid L, Siddiqi SA, khan RM, Ghauri I, Sheikh I. Upper limb musculoskeletal abnormalities in type 2 diabetic patients in low socioeconomic strata in Pakistan. BMC Research Notes 2013;6(16):1-6.

14. Barbara Dipiro: Pharmacotherapy A Pathophysiologic Approach. $7^{\text {th }}$ edition 2008:1205-1241,1505-1519.

15. Wyatt LH, Ferrance RJ. The musculoskeletal effects of diabetes mellitus. J Can Chiropr Assoc 2006;50(1):43-50. 